\title{
Measurement of the Hall current density in a Corbino geometry 2D electron gas
}

\author{
S. A. J. Wiegers \\ High Field Magnet Laboratory and Research Institute for Materials, University of Nijmegen, Postbus 9010, NL-6500 GL Nijmegen, \\ The Netherlands \\ and Grenoble High Magnetic Field Laboratory, MPI-CNRS, Boîte Postale 166, F-38042 Grenoble Cedex 9, France \\ J. G. S. Lok and M. Jeuken \\ High Field Magnet Laboratory and Research Institute for Materials, University of Nijmegen, Postbus 9010, NL-6500 GL Nijmegen, \\ The Netherlands \\ U. Zeitler \\ High Field Magnet Laboratory and Research Institute for Materials, University of Nijmegen, Postbus 9010, NL-6500 GL Nijmegen, \\ The Netherlands \\ and Institut für Festkörperphysik, Universität Hannover, Appelstrasse 2, D-30625 Hannover, Germany \\ J. C. Maan \\ High Field Magnet Laboratory and Research Institute for Materials, University of Nijmegen, Postbus 9010, NL-6500 GL Nijmegen, \\ The Netherlands \\ M. Henini \\ Department of Physics, University of Nottingham, Nottingham NG7 2RD, United Kingdom
}

(Received 4 December 1998)

\begin{abstract}
We have investigated the distribution of the Hall current in a Corbino geometry two-dimensional electron gas (2DEG) under quantum Hall effect conditions by measuring the induced magnetic moment. The magnetic moment near integer filling factor shows oscillations around the quantized value, which reflect a spatial redistribution of the Hall current density. The results show that at integer filling factor the local Hall conductivity in a high mobility sample is not quantized. Between integer filling factor the normal Hall current with the expected magnitude $V_{r} \nu e^{2} / h$ is measured, showing the reliability of the technique. [S0163-1829(99)03911-9]
\end{abstract}

Since the discovery of the quantum Hall effect, there have been several experimental and theoretical investigations to determine the local current distribution when an integer number of Landau levels is filled. Inductive techniques ${ }^{1-5}$ have shown that bulk states are able to carry a substantial amount of Hall current in the quantized region, although the bulk Hall conductivity $\sigma_{x y}$ could not be unambiguously determined. In particular, the question whether a fully quantized Hall current can be supported exclusively by bulk states remains experimentally unanswered. Theoretical work has indeed shown that the charge distribution and Hall field at the quantum Hall plateaus may not be homogeneous. ${ }^{6-8}$

In this paper, we address the question of the current distribution using a technique based on measuring the induced magnetic moment of the azimuthal dc Hall current in a Corbino two-dimensional electron gas (2DEG) driven by an applied radial voltage $V_{r}$. In a Corbino sample in a highmagnetic field the current flows at the Hall angle with respect to the radial-electric field and effectively will flow many times around the inner contact before reaching the outer contact. The largest Hall current density will occur in that part of the sample where the radial conductivity $\sigma_{x x}$ is smallest (Hall angle closest to $90^{\circ}$ ), and as a result the contribution to the Hall current coming from the highly resistive interior of the 2DEG will dominate over any edge channel contribution. The induced magnetic moment, which is measured with a sensitive torque magnetometer, ${ }^{9}$ depends on the current distribution in the $2 \mathrm{DEG}$.
The sample consists of a square $5 \times 5 \mathrm{~mm}^{2}$ piece of GaAs- $\mathrm{Al}_{x} \mathrm{Ga}_{1-x} \mathrm{As}$ heterostructure onto which two circular $\mathrm{Ni}_{x} \mathrm{Au}_{1-x} \mathrm{Ge}$ contacts are diffused to form a Corbino geometry. The solid inner contact has $0.5-\mathrm{mm}$ outer radius $R_{1}$ and the outer ring-shaped contact has $2.0-\mathrm{mm}$ inner radius $R_{2}$. The electron density and mobility at $4.2 \mathrm{~K}$ are, respectively, $1.4 \times 10^{11} \mathrm{~cm}^{-2}$ and $0.6 \times 10^{6} \mathrm{~cm}^{2} / \mathrm{Vs}$ and after illumination with a light emitting diode (LED) increase to, respectively $2.6 \times 10^{11} \mathrm{~cm}^{-2}$ and $1.0 \times 10^{6} \mathrm{~cm}^{2} / \mathrm{Vs}$. A pair of thin twisted $\mathrm{Cu}$ lead wires are attached to the contacts with some silverpaint. The sample is mounted in the torque magnetometer ${ }^{9}$ with the normal to the $2 \mathrm{D}$ plane making a $30^{\circ}$ angle with the magnetic field. The magnetization signal is calibrated using a 10 turn coil glued to the sample platform.

To obtain the torque $\mathbf{M} \times \mathbf{B}$ due to the Hall current in the 2DEG, the magnetometer signals from field sweeps with the magnetic field reversed are summed and their respective signals at zero applied voltage are subtracted. In this way, the lead wire contribution, which changes sign upon field reversal, and the background magnetizations of both the magnetometer and the sample are eliminated. By taking the difference of signals with reversed field direction the wire contribution, which is proportional to $I_{r} B$, is obtained.

Data taken from measurements with constant applied voltage are shown in Fig. 1. The induced torque is seen to be field independent, except near integer filling factor where it shows oscillations around a constant value. 

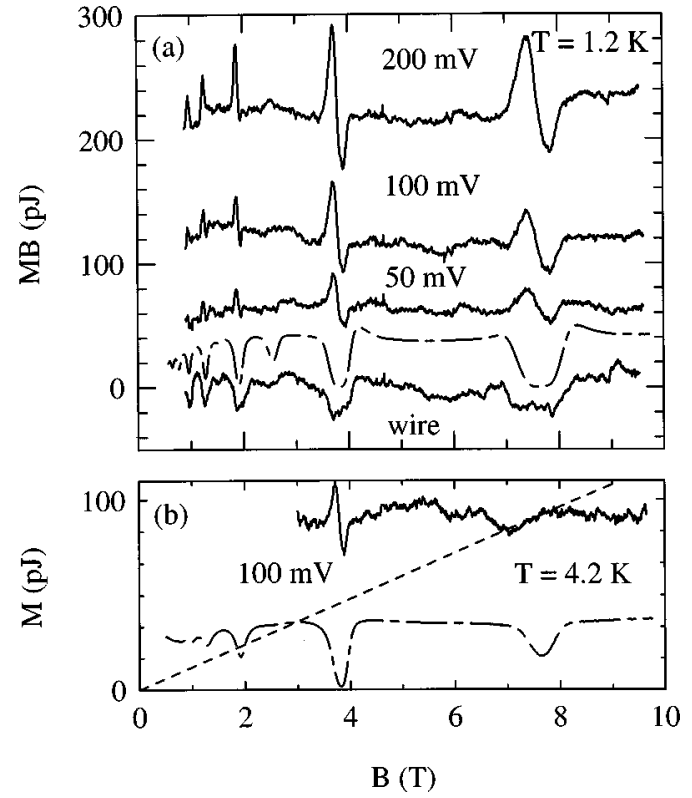

FIG. 1. The torque due to the Hall current for several values of $V_{r}$ before illumination where $\nu=1$ corresponds to $B=7.7 \mathrm{~T}$; (a) at $T=1.2 \mathrm{~K}$. The lead wire contribution to the torque and the product $\sigma_{x x} B$ (dashed) of the radial conductivity and the magnetic field are seen to be proportional to each other; (b) at $T=4.2 \mathrm{~K}$. The torque due to the Hall current for $V_{r}=100 \mathrm{mV}$ and the product $\sigma_{x x} B$ (dashed) before illumination are shown. The significance of the dashed straight line is discussed in the text.

To understand the data we calculate the induced magnetic moment for a homogeneous current distribution, which implies a simple $r^{-1}$ dependence of the radial-electric field. The following relation between the induced magnetic moment $M_{z}$ and the bulk $\sigma_{x y}$ can be obtained

$$
M_{z}=\frac{1}{2} \sigma_{x y} V_{r} S \ln ^{-1}\left(\frac{R_{2}}{R_{1}}\right)=\pi^{-1} \frac{\sigma_{x y}}{\sigma_{x x}} I_{r} S,
$$

where $S$ is the surface area between the two circular contacts and the two equalities hold, respectively, for constant applied voltage $V_{r}$ and current $I_{r}$. Since $\sigma_{x y}$ is proportional to $B^{-1}$, a constant torque in Fig. 1 is expected according to the first part of Eq. (1), which connects our results to normal Hall bar measurements. ${ }^{10}$ Figure 2 shows $\sigma_{x y}^{-1}$ obtained using Eq. (1) and the results presented in Fig. 1. These curves exhibit the linear behavior of the Hall effect at noninteger filling factor and the overall slope of the Hall curves is as can be expected on the basis of Eq. (1) and the sample parameters within the uncertainty of about $20 \%$ of the torque calibration.

Therefore, we conclude that away from integer filling factor the Hall current is a bulk current with a density proportional to $r^{-1}$ and $\sigma_{x y}$ is given by $\nu e^{2} / h$. These conclusions could not be drawn from previous measurements, although experiments using induction, ${ }^{2,5}$ as well as experiments on the Hall voltage distribution with electrodes in the interior of the 2DEG (Refs. 11-13) are in agreement with our observation of a bulk current.

We now turn to the behavior of $M_{z}$ near integer $\nu$ at $T$ $=1.2 \mathrm{~K}$ and remark that the oscillations observed at $T=1.2$

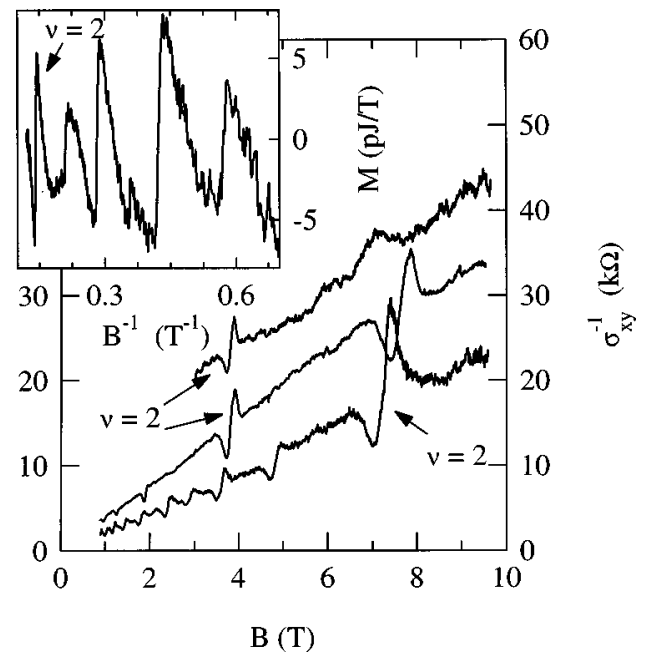

FIG. 2. $\sigma_{x y}^{-1}$ as obtained using Eq. (1). The upper curve is at $T=4.2 \mathrm{~K}$ before illumination and has been shifted by $+10 \mathrm{k} \Omega$ for clarity. The middle curve is at $T=1.2 \mathrm{~K}$ before illumination and the lower curve is at $T=1.2 \mathrm{~K}$ after illumination. The inset shows the equilibrium magnetization of the 2DEG at $T=1.2 \mathrm{~K}$ after illumination with zero applied voltage.

$\mathrm{K}$ in Figs. 1(a) and 2 do not reflect a homogeneous Hall current density and a plateau in $\sigma_{x y}$. A plateau in $\sigma_{x y}$ would correspond to a torque falling on a linear curve passing through the origin, shown in Fig. 1(b). This behavior requires an initial decrease and subsequent rise of the torque, as is only observed at $T=4.2 \mathrm{~K}$ for $\nu=1$, where $\sigma_{x x}>0$, but not at $T=1.2 \mathrm{~K}$ where $\sigma_{x x} \sim 0$. We believe that the oscillations are caused by a field-dependent, inhomogeneous Hall current density in the bulk of the 2DEG and not by variations in the total Hall current, which would imply a serious loss of quantization near integer filling factor.

To prove that the observed oscillations are also not connected to breakdown of the quantum Hall effect, we note that the torque signals in Fig. 1(a) are independent of the applied dc voltage up to at least $100 \mathrm{mV}$. Furthermore, we have checked that the widths of the radial resistivity minima do not vary (within 5\%) for voltages in the range 10 to $100 \mathrm{mV}$. If we compare these numbers to the Hall voltage in a Hall bar geometry, we see that our excitation voltages would correspond to injected currents of less than $100 \mathrm{nA}$ across a several $100-\mu \mathrm{m}$ wide Hall bar, which are acceptable values. Furthermore, typical values for breakdown voltages in much smaller Corbino disks of about $100-\mu \mathrm{m}$ wide have been found to be of order $1 \mathrm{~V} .{ }^{14}$ Our applied voltages are therefore about two orders of magnitude below the breakdown threshold.

To corroborate this point, we also performed measurements with a constant applied $I_{r}$. In principle such an experiment is not a good probe of the almost zero-current state near integer filling factor, but it does allow us to confirm the reliability of the experimental data at constant voltage. In addition, the radial current is injected across a rather large (1-mm inner diameter and 1.5-mm wide) Corbino disk, keeping the current density relatively small. We show in Fig. 3 the magnetic moment and the measured voltage across the 2DEG near $\nu=2$ at $T=1.2 \mathrm{~K}$. Using Eq. (1) it can be seen that in the constant current mode $M_{z} / V_{r}$ is proportional to 


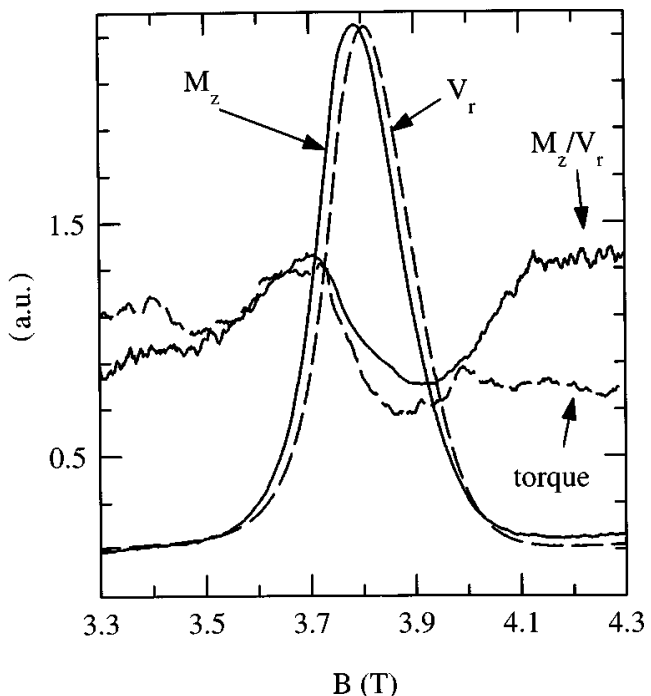

FIG. 3. The magnetic moment of the Hall current near $\nu=2$ when $I_{r}=1 \mu \mathrm{A}$, and the induced radial voltage $V_{r}$ (dashed), which has been scaled such that the maxima of the two peaks coincide. For comparison the ratio $M_{r} / V_{r}$ and the corresponding torque at constant voltage (dashed) are also shown. All curves are at $T$ $=1.2 \mathrm{~K}$ before illumination.

$\sigma_{x y} S \ln ^{-1}\left(R_{2} / R_{1}\right)$, since both $M_{z}$ and $V_{r}$ contain the factor $\sigma_{x x}^{-1}$. Therefore, $M_{z} / V_{r}$ has been plotted as well in Fig. 3 and it is clear that the results are fully equivalent to the constant voltage measurements. Since the excitation voltage varies substantially at constant current, these results indicate that breakdown effects are not relevant to our data.

As a final consistency check we note that the wire contribution to the observed torque in Fig. 1 is proportional to the product of the $\sigma_{x x}$ and the magnetic field $B$, and Fig. 2 shows that the magnitudes of $\sigma_{x y}$ at equal filling factor but different density are the same within experimental accuracy.

To obtain information on the importance of density inhomogeneities in our sample, we have measured the de Haasvan Alphen magnetization, shown in the inset to Fig. 2, of the same 2DEG sample with wires and contacts but without applied voltage. We observe a sawtooth-like magnetization, with magnetization jumps at both even and odd filling factors, characteristic for an uncontacted, high mobility 2DEG. ${ }^{15}$ This is an important result, since it shows that the contacts and wires connected to the 2DEG do not significantly disturb the density and the potential landscape at the edges. The field width of the magnetization jumps is a measure for the degree of disorder and it corresponds to at most a 5\% density variation across the sample.

Our experimental results can be summarized as follows. $M_{z}$ in the Corbino geometry reflects $\sigma_{x y}$ accurately between Hall plateaus. However, $M_{z}$ does not show the quantized value of $\sigma_{x y}$ at integer filling, as naively expected, but instead exhibits a sizable oscillation. Experimentally, inhomogeneities in the sample are found to be small even in the presence of wires and contacts, while nonlinearities in the current (breakdown) do not occur.

These results clearly point to a macroscopic redistribution of the Hall current going back and forth between inner and outer contact as we sweep through integer filling factor. This type of change in the current distribution would indeed lead to a larger magnetic moment when the highest carrier density is at the outer edge and smaller at the inner edge. To estimate the magnitude of such an effect we may assume that the highly resistive state of the sample is nucleated near the contacts and moves in a ringlike structure from outside to inside as the field in increased. Consequently, the radial voltage drop and the Hall current density will be largely localized within this region. Taking the extreme case that all of the current is moving from the outside to the inside, the geometrical factor $S \ln ^{-1}\left(R_{2} / R_{1}\right)$ increases from $\pi\left(R_{2}^{2}\right.$ $\left.-R_{1}^{2}\right) \ln ^{-1}\left(R_{2} / R_{1}\right)$ (away from integer filling) to $\pi R_{2}^{2}$ (beginning of the plateau), decreases to $\pi R_{1}^{2}$ (at the end of the plateau), and finally increases back to the initial value. We obtain extremal values of about 3.0 and 0.19 times the expected $M_{z}$ assuming a homogeneous current. Experimentally, the variations are in the range of 1.3 to 0.8 , showing that the Hall current redistribution is much more modest. However, this model does qualitatively describe our results.

The field range of the fluctuations in $M_{z}$ coincides with the range where the diagonal conductance approaches zero. This result indicates that the Hall current deviates as soon as integer filling factor is reached in some part of the Corbino ring, leading to a zero-radial conductance of the whole device. Indeed, the fluctuation at $\nu=2$ persists in the measurements at $T=4.2 \mathrm{~K}\left(\sigma_{x x} \approx 0\right)$, but near $\nu=1$, where the minimum of $\sigma_{x x}$ is much less pronounced, we find the expected behavior for the torque, with a plateau in $\sigma_{x y}$. It is also noteworthy that $M_{z}$ deviates from the expected values only within the field region where the equilibrium magnetization displays a jump, showing that the small inhomogeneity indicated by the sharp magnetization jump already causes a substantial Hall current density redistribution. This result demonstrates how sensitive the bulk Hall current distributions to sample imperfections, a fact considered to be a conditio sine qua non to observe the quantum Hall effect (QHE) ${ }^{10}$ Our results thus clearly show that the local Hall current is not quantized when the 2DEG becomes insulating under QHE conditions.

This conclusion is in line with earlier observations done with contacts in the interior of the 2DEG (Refs. 11-13) or with ac experiments measuring the radial Hall current across a loaded Corbino device. ${ }^{2}$ However, our experiment measures equilibrium properties and does not involve any interior contacts.

We would like to point out that the picture of a local filling factor resembles the description of the integer quantum Hall effect (IQHE) in terms of a percolation transition, ${ }^{6}$ in which the electron states fall in two distinct classes: local fibers and global fibers. Whereas global fiber states encircle the central contact and contribute to the total Hall current, local fibers encircle a point within the Corbino ring and cause an inhomogeneous Hall current density, but do not contribute to the total Hall current. While in this theory ${ }^{6}$ the total Hall current exhibits quantization, its distribution over the sample interior is inhomogeneous and varies with magnetic field. This picture is at least in qualitative agreement with our experimental results.

Summarizing, we have obtained a clean measurement of the Hall current distribution of a 2DEG in a Corbino geometry by measuring the associated magnetic moment with a 
sensitive torque magnetometer. We observe the expected homogeneously distributed Hall current in between integer filling factors, but a significant redistribution of the Hall current near integer filling factor at low temperatures. We conclude that local quantization of the Hall current does not occur in our experiment, although the total Hall current is quantized.
We thank A.K. Geim, A.G.M. Jansen, and N. Gauss for stimulating discussions. We are grateful to A.S. van Steenbergen for his expert advice on torque magnetometry. This work was supported in part by "De Stichting FOM," which in turn is financially supported by NWO, and by the European Community under Contracts Nos. 658768 and ERBFMGECT950077 through the TMR program.
${ }^{1}$ D.A. Syphers, K.P. Martin, and R.J. Higgins, Appl. Phys. Lett. 48, 293 (1986).

${ }^{2}$ P.F. Fontein, J.M. Lagemaat, J. Wolter, and J.P. André, Semicond. Sci. Technol. 3, 915 (1988).

${ }^{3}$ V.T. Dolgopolov, A.A. Shashkin, N.B. Zhitenev, S.I. Dorozhkin, and K. von Klitzing, Phys. Rev. B 46, 12560 (1992).

${ }^{4}$ B. Jeanneret et al., Phys. Rev. B 51, 9752 (1995).

${ }^{5}$ E. Yahel, D. Orgad, A. Palevski, and H. Shtrikman, Phys. Rev. Lett. 76, 2149 (1996).

${ }^{6}$ R.F. Kazarinov and S. Luryi, Phys. Rev. B 25, 7626 (1982); S. Luryi and R.F. Kazarinov, ibid. 27, 1386 (1983).

${ }^{7}$ D. J. Thouless, Phys. Rev. Lett. 71, 1879 (1993).

${ }^{8}$ J.J. Palacios and A.H. MacDonald, Phys. Rev. B 57, 7119 (1998), and references therein.

${ }^{9}$ S.A.J. Wiegers et al., Rev. Sci. Instrum. 69, 2369 (1998).

${ }^{10}$ See, e.g., The Quantum Hall Effect, edited by R.E. Prange and S.M. Girvin (Springer-Verlag, New York, 1987).

${ }^{11}$ H.Z. Zheng, D.C. Tsui, and Albert M. Chang, Phys. Rev. B 32, 5506 (1985).

${ }^{12}$ E.K. Sichel, H.H. Sample, and J.P. Salerno, Phys. Rev. B 32, 6975 (1985).

${ }^{13}$ G. Ebert, K. von Klitzing, and G. Weimann, J. Phys. C 18, L257 (1985).

${ }^{14}$ M. Yokoi et al., Physica B 249-251, 93 (1998).

${ }^{15}$ S.A.J. Wiegers et al., Phys. Rev. Lett. 79, 3238 (1997). 